\title{
Erratum to: Evaluation of the quality of CT images acquired with the single energy metal artifact reduction (SEMAR) algorithm in patients with hip and dental prostheses and aneurysm embolization coils
}

\author{
Akinaga Sonoda $^{1} \cdot$ Norihisa Nitta $^{1} \cdot$ Noritoshi Ushio $^{1} \cdot$ Yukihiro Nagatani $^{1} \cdot$ \\ Noriaki Okumura $^{2} \cdot$ Hideji Otani $^{1} \cdot$ Kiyoshi Murata $^{1}$
}

Published online: 14 October 2015

(C) Japan Radiological Society 2015

\section{Erratum to: Jpn J Radiol}

DOI 10.1007/s11604-015-0478-2

The authors' affiliations appeared incorrectly in the article cited above. The authors' correct affiliations are given here.

The online version of the original article can be found under doi:10.1007/s11604-015-0478-2.

Akinaga Sonoda

akinaga@belle.shiga-med.ac.jp

Norihisa Nitta

r34nitta@yahoo.co.jp

Noritoshi Ushio

ushio@belle.shiga-med.ac.jp

Yukihiro Nagatani

yatsushi@belle.shiga-med.ac.jp

Noriaki Okumura

noriaki@belle.shiga-med.ac.jp

Hideji Otani

otani@belle.shiga-med.ac.jp

Kiyoshi Murata

murata@belle.shiga-med.ac.jp

1 Department of Radiology, Shiga University of Medical

Science, Setatsukinowa-cho, Otsu, Shiga 520-2192, Japan

2 Department of Orthopedic Surgery, Shiga University

of Medical Science, Setatsukinowa-cho, Otsu, Shiga

520-2192, Japan 